\title{
Validation of the General and Personal Belief in a Just World scales in Iran and Their Relations to Perceived Threat of Kin Favoritism
}

\author{
Mehdi Mikani $^{1}$, Parisa Rafiee ${ }^{2}$, and Matthias Donat ${ }^{3}$ \\ Tarbiat Modares University ${ }^{1}$ \\ University of Rhode Island ${ }^{2}$ \\ Martin Luther University of Halle-Wittenberg ${ }^{3}$
}

\section{Author Note}

\section{Statements and Declarations}

The authors received no specific funding for this work.

The authors declare that they have no conflict of interest.

All applicable international and national ethical guidelines were followed.

The datasets generated during and/or analysed during the current study are available in OSF, https://osf.io/rdhu5/?view_only=7cb135327e9649559d94ebdfac449633

(C2021, American Psychological Association. This paper is not the copy of record and may not exactly replicate the final, authoritative version of the article. Please do not copy or cite without authors' permission. The final article will beavailable, upon publication, at [pending].

Corresponding author email: mahdimiki74@gmail.com

Mehdi Mikani (D) https://orcid.org/0000-0001-7523-1426 


\begin{abstract}
People possess a pre-conscious need to believe in the existence of justice in the world. This belief in a just world (BJW) is usually measured with self-report scales. Dalbert et al. (1987) and Dalbert (1999) have developed the general belief in a just world (GBJW) and personal belief in a just world (PBJW) scales as psychometrically robust measures of just-world beliefs. We conducted three studies to demonstrate the validity of the Persian versions of belief in a just world scales and the importance of distinguishing between GBJW and PBJW. First, we confirmed the factor structure. reliability, convergent validity (self-esteem, life satisfaction, and religiosity), and divergent validity (big five personality factors and dark triad traits) of GBJW and PBJW using Iranian participants $(N 1=454)$. Second, the associations of GBJW and PBJW with perceived threat of unjust behavior directed to self or others were assessed using two scenario-based studies $($ N2 $=81$, N3 $=$ 71) in the context of kin favoritism. While GBJW negatively predicted perceived threat of kin favoritism directed to others, PBJW was a negative predictor of perceived threat of kin favoritism directed to self. Consistent with theoretical assumptions, perceived likelihood of punishment mediated these associations. In addition to providing further evidence for the differentiation between GBJW and PBJW, the findings of Study 2 and 3 also support the idea of an optimistic bias towards self by showing that people perceived less threat of kin favoritism when others, rather than themselves, were potential victims of injustice.

Keywords: Belief in a just world, General belief in a just world, Personal belief in a just world, Kin favoritism, Discrimination, Perceived injustice
\end{abstract}




\section{Validation of the General and Personal Belief in a Just World scales in Iran and Their Relations to Perceived Threat of Kin Favoritism}

\section{Introduction}

Despite the famous figure of speech, people do not always "reap what they sow." Humanity suffers from varieties of injustice such as world hunger and wars or everyday discrimination against women as well as racial, religious, and sexual minorities. Yet according to one of the most influential theories about human belief systems, people adopt just-world beliefs that serve to enable them to construe their social environment as if it were just, stable, and orderly (Lerner, 1980). Furthermore, these beliefs provide people with certain cognitive ways to justify the status quo, underestimate the prevalence of injustice, or even blame innocent victims. Given these functions, just-world beliefs have been found to be an important factor in well-being, religion, and justice-related perceptions or behavior. Despite the prevalence and importance of just-world beliefs, rigorous research on the psychology of these beliefs is lacking in Iran. To help overcome this gap, we aimed to validate self-report measures of individual differences in just-world beliefs, and test their correlates and predictive implications. As a common and understudied justice-related issue that many people, especially in Iran, are confronted with on a daily basis takes root in favoritism toward kinship, we focus on perceptions of kin favoritism as a part of investigating the validity of just-world belief scales.

\section{Belief in a just world}

According to just-world theory, many people across the world believe in a just world where people get what they deserve and deserve what they get. The BJW is considered a personality trait with an importance in the interaction between personality and other areas of social science. There are some adaptive functions that BJW is thought to serve, such as the confidence that important decisions about people are made with justice and the perception of life as safe and structured. In line with this idea, BJW has been found to be 
associated with self-esteem (Donat et al., 2016, Tatsi \& Panagiotopoulou, 2021), life satisfaction (Dalbert, 1999), and a decrease in the perception of discrimination and unfairness of others (Furnham, 2003). It is essential to consider that while an individual can be an observer who only judges and interprets the world, the individual can also act as a participant. Therefore, Lipkus et al. (1996) propose that a general belief in a just world (GBJW) should be distinguished from a personal belief in a just world (PBJW).

The general and personal belief in a just world scales were developed to measure the explicit endorsement of just-world beliefs (Dalbert, 1999, Dalbert et al., 1987). GBJW refers to the confidence that people, in general, get what they deserve and deserve what they get. On the other hand, PBJW refers to the confidence that a person is usually treated in a just manner and plays an important adaptive role for people.

GBJW and PBJW were found to be positively correlated, however, they are identified with different meanings and psychological functions (see Dalbert, 1999; Lipkus et al., 1996, Nartova-Bochaver et al., 2018; Sutton \& Douglas, 2005). GBJW helps people maintain subjective well being (Dalbert, 1999), minimize the perceived injustices (Sutton \& Douglas, 2005), and defend against threats to just-world beliefs by strategies such as victim blaming (Hafer \& Gosse, 2011). PBJW positively relates to individuals' forgiveness (Strelan \& Sutton, 2011), trust in others (Cubela Adoric \& Kvartuc, 2007; Yu et al., 2020), and different aspects of subjective well-being (Dalbert, 1999; Donat et al., 2016). In attempting to examine the distinction between GBJW and PBJW we will explore people's trust in justice.

\section{BJW, trust in justice, and the differentiation of GBJW and PBJW}

As noted earlier, a function of both GBJW and PBJW is that it could shape individuals' trust in others or the perceived risk of becoming a victim of unfair decisions (Bai et al., 2014; Cubela Adoric \& Kvartuc, 2007; Hafer \& Gosse, 2011). To the extent that individuals believe the world treats others fairly (GBJW), they are less likely to perceive 
injustice as prevalent and anticipate that others will not become a victim of injustice. In a similar fashion, individuals' belief about how they themselves are treated by the world (PBJW) is linked to a prediction of outcomes in situations where they are the potential victims of injustice. In this article, we leverage the above mentioned arguments to validate BJW scales and also look at the differentiation between GBJW and PBJW.

Injustice can take many forms among which there is kin favoritism. Kin favoritism is a common type of injustice that arises from people's preferences to favor members of their own extended family at the expense of injustice to outsiders. Such favoritism is especially prevalent in societies that have large close-knit family groups and strong family ties, such as Iran (Akbari et al., 2020). Thus, we focus on kin favoritism to investigate individuals' perceived threat of injustice.

Next, we go beyond examining the mere association between BJW and perceived threats and take the further step of identifying the underlying process which mediate the relationship. First, BJW has been linked with anticipating a high possibility of punishment (Bai et al., 2014, 2016). This link stems from a fundamental premise of BJW, which is that good behaviors are rewarded and bad behaviors are punished. Second, perceived risk of punishment is a factor that people consider when they estimate the threat and prevalence of injustice. This relation occurs because, intuitively, people tend to engage in less illegal behaviors when the risk of punishment is high. Taken together, believing in a just world indicates the extent to which individuals believe that people who do wrong will get punished which in turn leads to the estimation of the likelihood that injustice takes place (Bai et al., 2014). While legal punishment is a central deterrent, the possibility of moral punishment and negative judgments of others can also relate to people's perceived threat of injustice. Thus, the role of the perceived risk of moral and legal punishment in the relationship between BJW and the perceived threat of kin favoritism is worthy of investigation.

In summary, we expect that GBJW, compared to PBJW, is more strongly 
associated with the perceived threat of injustice (kin favoritism in this case) and punishment when the person is a mere witness of potential injustice. In contrast, when the person is the unfortunate target of unfair treatment, PBJW is expected to be more strongly associated with the anticipation of outcomes. Moreover, perceived likelihood of punishment is expected to explain the relationship between BJW dimensions and the perceived threat of kin favoritism.

While our focus in this paper is validating BJW scales and testing their predictive implications, a trace of optimistic bias can be found in the investigation of injustice threats. Literature on optimistic bias suggests that people tend to assume that they are more likely, compared to similar others, to avoid an undesirable outcome or reach a favorable one (Shepperd et al., 2013, Strelan \& Callisto, 2020). Thus, we briefly analyze the optimistic bias in the context of kin favoritism in Iran, as we discuss shortly, individuals are expected to predict that others are more likely than themselves to become victims of kin favoritism.

\section{BJW and well-being}

The just-world hypothesis states that people who believe that the world treats them fairly may be confident in planning for long-term future goals, expect their lives to be orderly, meaningful, and controllable. Directly or indirectly mediated by these implications, mental health is improved (Kiral Ucar et al., 2019). Indeed, previous research on BJW's effects on well-being suggests that both GBJW and PBJW are associated with self-esteem (i.e. high self-esteem reflects a primary feeling of self-worth and self-admiration, while low self-esteem represents unhappiness with oneself, self-denial, and lack of self-respect), life satisfaction, and other components of well-being (Donat et al., 2016. Nartova-Bochaver et al., 2019, Tatsi \& Panagiotopoulou, 2021). Also, the literature emphasizes that PBJW is a stronger predictor of these constructs. 


\section{BJW and religiosity}

Religious teachings promote the ideas that sufferings will be compensated and good deeds will ultimately be rewarded. Furthermore, several religious traditions and teachings across different religious affiliations, especially karmic religions, provide a supernatural framework in which actions are expected to lead to valence-congruent outcomes, meaning that mean people experience misfortune, and nice people experience success (Harvey \& Callan, 2014; White et al., 2019). This idea reflects just-world believers' conviction that-in a just world-good things happen to good people and bad things happen to bad people. The literature also generally supports a positive relationship between BJW and religiosity (Bègue, 2002, Harvey \& Callan, 2014).

\section{BJW and personality}

BJW and the Big Five personality factors represent divergent constructs conceptualized as assumptions about justice in the world and self-describing attributes, respectively. Accordingly, Big Five personality factors can be used for testing the divergent validity of BJW scales. Although the two constructs are broadly considered as differentiable constructs and the Big Five items do not include the words "fair" or "just",

researchers found inconsistent results about the associations of GBJW and PBJW with the Big Five factors (Bollmann et al., 2015; Nudelman, 2013). Previous studies investigating these associations did not report any notable difference in the strength of the correlations with regard to GBJW and PBJW. Consistent with the literature (Bollmann et al., 2015 , Nudelman, 2013), we expect that both BJW dimensions will have weak positive associations with extraversion and emotional stability, and nonsignificant associations with agreeableness, conscientiousness, and openness to experience.

Dark Triad traits (i.e. narcissism, psychopathy, and Machiavellianism) are characterized by exploiting others, dishonesty, aggressiveness, duplicity, and emotional coldness (Paulhus \& Williams, 2002). We argue that antisocial people do not need to form 
a specific assumption about justice in the world as they may not care for justice and fairness at all. However, one might argue that BJW has been negatively correlated with rule-breaking behavior and antisocial tendencies (e.g., Donat et al., 2012). Thus, we argue that dark triad traits could be used for divergent validity as we expect that there will be weak negative relationships between BJW dimensions and the dark triad traits, if any. Consistent with this assumption, previous research found no significant associations between BJW and narcissism (Ames et al., 2006) as well as honesty-humility personality factor (Bollmann et al., 2015).

\section{The present studies}

The aim of the present set of studies is two-fold. In study 1, a principal component analysis (PCA) and a confirmatory factor analysis (CFA) were done on the Persian version of the GBJW and PBJW in order to investigate whether the two-factor structure, as reported in other cultures, can be supported in the Iranian context. Furthermore, the convergent and divergent validity of the Persian BJW scales were examined. For convergent validity, we tested the correlations of BJW scales with self-esteem, life satisfaction, and religiosity. For divergent validity, we examined the correlations of BJW scales with Big Five personality factors (Extraversion, Agreeableness, Conscientiousness, Emotional Stability, and Openness) as well as Dark Triad traits (Narcissism, Psychopathy, and Machiavellianism). More specifically, we hypothesize that both GBJW and PBJW will be positively associated with life satisfaction, self-esteem, and religiosity, but negatively associated with dark triad traits. Moreover, Big Five factors will be unrelated or weakly associated with GBJW and PBJW.

In study 2 and study 3, we aim to further test the validity of the scales by assessing the distinction between GBJW and PBJW in predicting the perceived threat of injustice and punishment directed at others (study 2) and themselves (study 3). Iran is a country with strong family ties and a large portion of close-knit family groups, and is ranked 149th 
in the corruption perception index (CPI, 2020). Kin favoritism is the form of injustice that we plan to investigate because kin-based favoritism is probably the most common type of corruption in Iran.

\section{Study 1}

In the first study, our aim was to test the psychometric properties of the Persian version of the General and Personal Belief in a Just World scales by using exploratory and confirmatory factor analysis, and examine the convergent and divergent validity of these scales.

\section{Method}

\section{Participants}

A total of 454 Iranian participants, aged between 17 and 65 years $(M=32.09$, $S D=10.628$ ), were recruited through online social media platforms such as Instagram, Twitter, etc. The sample consisted of $68.5 \%$ female and $31.5 \%$ male participants and the majority (59.5\%) were not married. Among the participants, 35 held a doctorate degree, 191 held a master's degree, 138 held a bachelor's degree and 91 had a diploma or less.

\section{Procedure and Measures}

After a brief introduction, the consenting participants completed an online questionnaire consisting of the General and Personal Belief in a Just World scales, as well as Rosenberg Self-esteem scale, Satisfaction with Life scale, Religiosity scale, Big Five scale, and the Dirty Dozen Dark Triad scale. Except for items in the religiosity scale, the extent to which the participants agreed with each statement was measured through a seven-point Likert scale that ranged from 1 (totally disagree) to 7 (totally agree).

Belief in a Just World. The General and Personal Belief in a Just World scales, the psychometric properties of which were identified by Dalbert (1999) and Dalbert et al. 
(1987), were translated into Persian through the method of translation-back translation. Participants are asked to indicate the degree to which they agree with the statements about general BJW (6 items) and personal BJW (7 items).

Self-esteem. We measured self-esteem with the Persian version of the 10-item Rosenberg Self-esteem Scale (RSES; Rosenberg, 1965). The RSES is commonly used to measure a person's overall self-esteem (e.g., "On the whole, I am satisfied with myself."). The Persian translation of this scale has shown adequate validity and reliability in previous work (Shapurian et al., 1987). The alpha coefficient of the RSES for this sample was .85.

Life Satisfaction. The 5-item Satisfaction with Life Scale (SWLS; Diener et al., 1985) was used to assess participants' global life satisfaction (e.g., "In most ways my life is close to my ideal."). The Persian version of the scale has shown adequate psychometric properties in previous research (Joshanloo \& Afshari, 2011). In our sample, Cronbach's alpha was .86.

Religiosity. Finally, participants responded to the 5-item Duke University Religion Index (DUREL; Koenig et al., 1997). The first two items measuring religious activities (e.g., "How often do you attend mosque or other religious meetings?") are rated along a 6-point scale ranging from 1 (never) to 6 (more than once a week/day). The last three items measuring intrinsic religiosity (e.g., "In my life, I experience the presence of God.") are rated along a five-point Likert-type scale ranging from 1 (definitely not true) to 5 (definitely true of me). The scores were transformed to $z$-scores since they have different response scales. The Persian version of this scale has shown very good psychometric characteristics (Hafizi et al., 2013). In the present study, the alpha coefficient of the scale was .89.

Big Five Personality. We measured the Big Five personality factors with the Ten-Item Personality Inventory (TIPI; Gosling et al., 2003) which is a very short measure of the Big Five. The TIPI consists of five subscales: Extraversion (e.g., "I see myself as: extraverted, enthusiastic."), Agreeableness (e.g., "I see myself as: sympathetic, warm."), 
Conscientiousness (e.g., "I see myself as: dependable, self-disciplined."), Emotional Stability (e.g., "I see myself as: calm, emotionally stable."), and Openness to Experience (e.g., "I see myself as: open to new experiences, complex."); each of them measured with two items. In the present study, internal consistency coefficients for extraversion, agreeableness, conscientiousness, emotional Stability, and openness to Experience were .77, .17, .53, .52, and .32, respectively. Despite being used extensively, estimates of the internal consistency

of the TIPI are low in different contexts (Ehrhart et al., 2009, Gosling et al., 2003; Romero et al., 2012). The Persian translation of this scale has been used in previous research in Iran (Atari \& Yaghoubirad, 2016).

Dark Triad Traits. The Dark Triad Dirty Dozen Scale (Jonason \& Webster, 2010) was used to measure narcissism (4 items; e.g., "I tend to seek prestige or status"), psychopathy (4 items; e.g., "I tend to lack remorse"), and Machiavellianism (4 items; e.g., "I tend to manipulate others to get my way"). The Persian translation of the 12-item questionnaire has shown satisfactory psychometric characteristics in previous research (Aghababaei et al., 2014). In the present study, internal consistency coefficients for narcissism, psychopathy, and Machiavellianism were .81, .23, and .73, respectively.

\section{Results}

In order to investigate the dimensional structure of the Persian BJW items, Principal Component Analysis (PCA) and Confirmatory Factor Analyses (CFA) were employed. First, the data sample was randomly split into two equal subsamples. The first subsample was used for PCA $(n=227)$ and the second subsample was used for CFA $(n=$ $227)$.

Principal component analysis. We evaluated the structure of the 13 items using a PCA with direct oblimin rotation. The Kaiser-Meyer-Olkin $(\mathrm{KMO}=0.902)$ and Bartlett's Test of Sphericity $(<0.001)$ indicated that our sample is adequate and suitable for PCA. The first principal component analysis showed two factors with eigenvalues 
greater than 1 (6.105 and 1.715), which was also confirmed by a Parallel and Scree Analyses as well. Consequently, a second PCA with direct oblimin rotation was performed with a fixed number of two factors. As can be seen in Table 1, all items of the personal belief in a just world formed the first factor, and all items of the general belief in a just world formed the second factor. This two-factor solution accounted for $60.15 \%$ of the total variance.

Consistent with previous studies (Dalbert, 1999), participants endorsed the PBJW more strongly than the GBJW, $M=4.38(S D=1.32)$ versus $M=3.50(S D=1.44), t(1$, $453)=-14.588, p<.001$. Also, age was positively correlated with GBJW $(r=.20, p<$ $.001)$, but not with PBJW $(r=.02, p=.532)$. However, there were no gender differences in GBJW (Welch $t$ statistic $=.623, p=.430)$ and PBJW (Welch $t$ statistic $=.315, p=.575)$.

Confirmatory factor analysis. We used CFA to examine the previously identified factor structure of the scale. Due to positive correlations between GBJW and PBJW, the two factors were permitted to co-vary. We assessed model fit using the Chi-square over degree-of-freedom ( $\left.\chi^{2} / \mathrm{df}\right)$, Goodness-of-Fit Index (GFI), the Comparative Fit Index (CFI), the Tucker-Lewis Index (TLI), the Root Mean Square Error of Approximation (RMSEA), and the Standardized Root Mean Square Residual (SRMR). Maximum likelihood was used as the estimation method. The analysis was performed using the lavaan statistical package in the $\mathrm{R}$ software environment.

In the first model, we assumed that the two-factor with a covariation between latent factors would account for the variance of 13 items. Similar to the Russian adaptation of just-world scales, our first model was not fit $\left(\chi^{2}(64)=217.504, \chi^{2} / \mathrm{df}=3.398\right.$; GFI $=$ $.872, \mathrm{CFI}=.890 ; \mathrm{TLI}=.866 ; \mathrm{RMSEA}=.103 ; \mathrm{SRMR}=.069)$. Following

Nartova-Bochaver et al. (2018)'s solution, we allowed the items with wording similarities that might share common variance to correlate (PBJW1 \& PBJW3, PBJW3 \& GBJW2, PBJW5 \& GBJW5, and GBJW3 \& GBJW5). The second model fit parameters reached values indicating good fit of the model $\left(\chi^{2}(64)=136.168, \chi^{2} / \mathrm{df}=2.127 ; \mathrm{GFI}=.921\right.$, CFI $=.945 ; \mathrm{TLI}=.929 ; \mathrm{RMSEA}=.075 ; \mathrm{SRMR}=.055)$. Internal consistency scores of GBJW 
( $\alpha=.85)$ and PBJW $(\alpha=.87)$ were high. To sum up, the two-dimensional structure of the Persian version of the BJW Scale and the distinction between GBJW and PBJW has been confirmed.

Convergent Validity. Self-esteem, life satisfaction, and religiosity were used to establish the convergent validity of the PBJW and GBJW scales. As presented in Table 2 , self-esteem is positively associated with PBJW and GBJW. Also, self-esteem was more strongly associated with PBJW than GBJW (Fisher's $\zeta=4.467, p<.001$ ). Results yielded significant associations between life satisfaction and both PBJW and GBJW. Similar to self-esteem, life satisfaction was more strongly associated with PBJW than GBJW (Fisher's $\zeta=4.172, p<.001$ ). Moreover, there was a positive correlation between religiosity and both dimensions of BJW. However, the association between religiosity and GBJW was stronger (Fisher's $\zeta=6.56, p<.001$ ).

Divergent Validity. To inspect the divergent validity of the BJW scales, we tested the correlations between PBJW and GBJW with the Big Five personality factors (Extraversion, Agreeableness, Conscientiousness, Emotional Stability, and Openness) and dark triad traits. The results indicated that GBJW was positively associated with extraversion, conscientiousness, and emotional stability . In a similar pattern, PBJW was positively associated with extraversion, conscientiousness, and emotional stability. However, the associations of the BJW dimensions with agreeableness and openness were not significant. Concerning the relations between dark triad traits and BJW dimensions, while Machiavellianism and narcissism were not significantly associated with BJW dimensions, there was a weak positive association of both GBJW and PBJW with psychopathy.

\section{Discussion}

The findings of this study confirm the two-dimensional structure of the Persian version of the BJW Scale, thus replicating the previous findings (Dalbert, 1999 . Nartova-Bochaver et al., 2018). Consistent with the literature (Dalbert, 1999; 
Nartova-Bochaver et al., 2018, Sutton \& Douglas, 2005), GBJW among Iranians was higher than PBJW. Regarding the relations between age and BJW dimensions, we found that older individuals believed more in GBJW than younger people. However, there was no significant relation between PBJW and age. Furthermore, in line with prior research (Nartova-Bochaver et al., 2018; Sutton \& Douglas, 2005), GBJW and PBJW scores were not different with respect to gender.

The significant positive relations between BJW dimensions and self-esteem as well as life satisfaction and religiosity demonstrate the convergent validity of GBJW and PBJW scales. Moreover, the stronger correlations of PBJW, compared to GBJW, with self-esteem and life satisfaction indicate that well-being may be more dependent on individuals' belief in being treated fairly by others rather than their belief in the existence of justice for people in general. Conversely, the stronger correlation of GBJW, compared to PBJW, with religiosity implies that religion may be more involved with the aspects of justice in society and for people in general. In addition to replicating previous research on BJW correlates (Bègue, 2002, Donat et al., 2016, Nartova-Bochaver et al., 2019), these finding support the different psychological functioning of GBJW and PBJW.

Our findings showed weak effect sizes or non-significant correlations of BJW dimensions with big five personality factors which partially confirm the divergent validity of BJW scales. However, as we will discuss in the limitations of this study, these findings should be interpreted cautiously due to low internal consistency of the scales. In line with previous studies (Nudelman, 2013), there were positive correlations of GBJW and PBJW with extraversion suggesting that believing in a just world may contribute to sociability and energetic behavior by reducing feelings of vulnerability. Furthermore, our findings on the relations between BJW dimensions and conscientiousness were consistent with Nudelman and Otto (2021)'s meta-analysis. We found positive correlations of both GBJW and PBJW with conscientiousness which demonstrates that just-world beliefs may be positively related to diligence, carefulness, and deliberation because believers in a just 
world are convinced that being conscientious will lead to deserved outcomes. Also, the positive associations between BJW dimensions and emotional stability were consistent with previous research (Nudemlan, 2013). This finding supports the notion that GBJW and PBJW are buffers against unfortunate incidents in one's life that decrease anxiety and depression (Furnham, 2003, Lipkus et al., 1996). Finally, agreeableness and openness did not have significant relationship with BJW dimensions which supports findings of previous research (Bollmann et al., 2015; Nudelman, 2013).

Regarding the relations with dark triad traits, there was no relationship between BJW dimensions and Machiavellianism as well as narcissism which further confirms the divergent validity of BJW scales. People high in Machiavellianism and narcissism may not simply care about justice and fairness, in turn they may not feel the need to form a particular view about the existence of justice in the world. Although psychopathy had weak positive correlations with GBJW and PBJW, this finding is unreliable because the internal consistency coefficient of psychopathy scale in this study was very low.

\section{Study 2}

In this study, we aim to test the relations of GBJW and PBJW with perceived threat of kin favoritism which participants witnessed as a potential injustice. This relation is expected to be mediated by perceived likelihood of punishment.

\section{Methods}

\section{Participants}

Participants were recruited from social media platforms (e.g., Twitter, Telegram) who were randomly assigned to study 2. Participants were 81 Iranians recruited from social media platforms (e.g., Twitter, Telegram), 35 males and 46 females who were between 24 and 58 years old $(M=32.57 ; S D=7.396)$. 


\section{Procedure and measures}

After reading the informed consent, participants first completed the GBJW and PBJW scales. Then, they read the designed scenarios and responded to measures of perceived likelihood of injustice and perceived likelihood of punishment. After completing the questionnaire, participants were thanked and fully debriefed. The contact information of the main researcher was provided in case participants had further questions. Full ethical review and approval were not required for this study in accordance with the national and institutional guidelines.

Belief in a just world. BJW was assessed with the Persian translation of the 6-item General Belief in a Just World and 7-item Personal Belief in a Just World Scale that were validated in study 1 . All responses were made on 7-point scales. The scores from the GBJW and PBJW items were averaged showing good internal consistency $(\alpha=.89$ and $\alpha=.88$, respectively). Higher scores indicated stronger endorsement of the GBJW and PBJW.

The kin favoritism scenarios. We designed five scenarios in a panel discussion among the authors and a social activist in justice-related issues. In these scenarios, three individuals were portrayed; a target of injustice, a favor receiver, and a favor provider. The favor provider could unfairly treat his/her kin favorably in each scenario which were in different contexts, including hiring for a government job and applying for an educational position. A sample scenario about hiring for a government job reads:

Two individuals have been chosen as the final candidates for a government job position. Considering the resumes, one of them is clearly better qualified for the job. A recruiter who works under the supervision of higher ranks has to do an interview with these candidates to decide who gets the job. Coincidentally, the recruiter turned out to be a relative of the less qualified candidate. This recruiter knows that discriminating between candidates and not choosing the most qualified person is against the rules.

Following each scenario, the perceived threat of kin favoritism was assessed by 
"please estimate the likelihood that the favor provider will favor/choose his/her kin" in a 9-point scales ranging from 1 (definitely will not) to 9 (definitely will). For the perceived likelihood of punishment, participants answered two questions; the likelihood of legal punishment and the likelihood of negative moral judgment by others. A sample item was "please estimate the likelihood of legal punishment from the favor provider's authorities if he/she favors his/her kin" on 9-point scales ranging from 1 (definitely will not be) to 9 (definitely will be). We averaged the scores of the five scenarios for the perceived threat of kin favoritism ( 5 items, $\alpha=.71$ ) and perceived likelihood of punishment (10 items, $\alpha=$ $.85)$.

\section{Results}

Table 3 shows the descriptive statistics and correlations of the variables in study. Correlations between GBJW, PBJW, perceived likelihood of punishment, and perceived threat of kin favoritism were significant in the expected directions.

Using the lavaan statistical package in the $\mathrm{R}$ software environment, a series of hierarchical regression analysis were performed to examine the effects of GBJW, PBJW, perceived punishment, and demographics on perceived threat of kin favoritism. As shown in table 4, we first entered age and gender as controlled variables followed by entering GBJW and PBJW in the second step. As expected, GBJW $(\beta=-.332, t=-2.718, p=$ $.008)$, but not PBJW ( $\beta=-.188, t=-1.541, p=.128)$ predicted perceived threat of kin favoritism. In the third step, perceived likelihood of punishment was included as a variable in the regression analysis and emerged as the only variable to significantly predict perceived treat of kin favoritism $(\beta=-.453, t=-4.714, p<.001)$. The effect of GBJW became non-significant after including perceived likelihood of punishment $(\beta=-.167, t=$ $-1.474, p=.145)$.

We further investigated the mediating effect of perceived likelihood of punishment in the relationship between GBJW and perceived threat of kin favoritism. The results 
showed that the indirect effect of GBJW on perceived threat of kin favoritism was significant $(\beta=-.136, p=.005)$. Indirect effects are not altered much when the PBJW is included as a covariate $(\beta=-.144, p=.009)$.

\section{Discussion}

The results of study 2 provide empirical support for the distinction between GBJW and PBJW and underscore the importance of considering this distinction in research on the functions of just-world beliefs in risk perception and trust in justice (Cubela Adoric \&

Kvartuc, 2007; Hafer \& Gosse, 2011). The findings demonstrate that when individuals are mere observers of potential unjust behavior, GBJW is central in their perceived risk of injustice. Furthermore, perceived likelihood of punishment mediated the relation between GBJW and the perceived threat of kin favoritism. Our findings are consistent with previous research on the relations between BJW and corruption perception (Bai et al., 2014).

\section{Study 3}

In the third study, we aim to test the relations of GBJW and PBJW with perceived threat of kin favoritism which participants imagined as a potential injustice directed to themselves. This relation is expected to be mediated by perceived likelihood of punishment.

In addition, we plan to assess whether the perceived risk of becoming a victim injustice is higher when the threat of unjust behavior is directed to self rather than others. To do so, the second and third studies were identically designed and the participants were randomly assigned to them.

\section{Methods}

\section{Participants}

The participants who were assigned to this study were 71 Iranians recruited from social media platforms (e.g., Twitter, Telegram). There were 33 males and 38 females 
between 23 and 48 years old $(M=30.52 ; S D=5.079)$.

\section{Procedure and measures}

The study was presented to participants as in Study 1. Participants were asked to read the informed consent before reading the scenarios and answering the questions.

Belief in a just world. GBJW and PBJW were assessed using previous studies' measures. The internal consistency for both GBJW and PBJW were .89.

The kin favoritism scenarios. The same five scenarios as study 2 were used with only a difference in the subject of injustice; participants imagined themselves as someone who is the unfortunate target of potential injustice. A sample scenario that participants read was:

"You have been chosen as one of the two final candidates for a government job position. Considering the resumes, you are clearly better qualified for the job than the other candidate. A recruiter who works under the supervision of higher ranks has to do an interview with you and the other candidate to decide who gets the job. Coincidentally, the recruiter turned out to be a relative of the other candidate. This recruiter knows that discriminating between candidates and not choosing the most qualified person is against the rules."

Following each scenario, the same items as study 2 for measuring perceived threat of kin favoritism and perceived likelihood of punishment were used. The scores of the five scenarios were averaged for the perceived threat of kin favoritism ( 5 items, $\alpha=.81$ ) and perceived likelihood of punishment (10 items, $\alpha=.87$ ).

\section{Results}

Table 5 shows the descriptive statistics and correlations of the variables in study 3 . There were significant correlations between GBJW, PBJW, perceived likelihood of punishment, and perceived threat of kin favoritism in the expected directions. 
We used the lavaan statistical package in the $\mathrm{R}$ software environment for regression and mediation analyses. Initially, a series of hierarchical regression analysis were conducted to investigate the effects of GBJW, PBJW, perceived likelihood punishment, and demographics on perceived threat of kin favoritism. As can be seen in table 6, we entered age and gender as controlled variables in first step before entering GBJW and PBJW in the second step. As expected, PBJW $(\beta=-.323, t=-2.319, p=.024)$, but not GBJW $(\beta$ $=-.137, t=-.982, p=.330)$ predicted perceived threat of kin favoritism. In the third step, perceived likelihood of punishment was included as a variable in the regression analysis and emerged as the variable to negatively predict perceived threat of kin favoritism $(\beta=-.367$, $t=-3.108, p=.003)$. The effect of PBJW became non-significant after including perceived likelihood punishment $(\beta=-.202, t=-1.480, p=.144)$.

Then, we further investigated the mediating effect of perceived likelihood of

punishment in the relationship between PBJW and perceived threat of kin favoritism. The results showed that the indirect effect of PBJW on perceived threat of kin favoritism was significant $(\beta=-.192, p=0.015)$ and did not change much when the GBJW was included as a covariate $(\beta=-.163, p=.036)$.

As the second and third studies were identical except for the potential victim of favoritism, we were able to test whether individuals perceive a higher level of injustice threat directed to others than themselves. According to results, participants in the first study $(M=6.26, S D=1.27)$ estimated a higher possibility of unjust behavior (Welch $t$ statistic $=8.493, p=.004)$ than participants in the second study $(M=5.55, S D=1.67)$.

\section{Discussion}

The analyses in study 3, provide further empirical evidence for the distinction between GBJW and PBJW. The findings of this study indicate that when individuals are the unfortunate targets of potential injustice, PBJW is the central dimension of the beliefs in a just world that relates to their perceived likelihood of injustice and punishment. 
Furthermore, perceived likelihood of punishment mediated the relation between PBJW and the perceived risk of becoming a victim of kin favoritism. Our findings are in line with the literature on the function of BJW in people's trust in others and risk perception (Bai et al., 2014; Cubela Adoric \& Kvartuc, 2007; Hafer \& Gosse, 2011).

In addition, our findings are of high importance because strong believers in a just world may strive to achieve their goals by just means because they trust in others' fairness (Č́abelková \& Hanousek, 2004). Therefore, BJW could further be a predictor of a person's own intentions for engaging in unjust behaviors (see Bai et al., 2016). Consistent with this idea, prior research has found that people with strong BJW are less likely to have illegal intentions and behaviors such as bribery (Bai et al., 2016, Dalbert, 1999) and engage in less bullying and cheating behavior at a young age (Donat et al., 2014; Donat et al., 2018).

Consistent with previous research on optimistic bias towards the self compared to others (for a review, see Shepperd et al., 2013), we found evidence of a bias for the perception of a negative outcome which was injustice victimization in the case of the present studies. Also, our findings to some extent support Strelan and Callisto (2020)'s claim that BJW is associated with the optimistic bias.

\section{Limitations}

There were some limitations in present studies. First, our samples mainly consisted of educated people living in Tehran which is the capital of Iran with a population of above-average education, economic status, and liberal political orientation. Thus, the results of our studies can be generalized to only some degree. Also, the second and third study have rather small sample sizes. Future research should replicate our findings in a more representative sample of Iranians with larger sample sizes.

A major limitation concerns our measures of the big five personality factors and dark triad traits. Big five personality dimensions were assessed using a short measure with two items for each factor. Given the brevity of the TIPI scales, it is not surprising that 
they usually have low alpha coefficients (Atari \& Yaghoubirad, 2016; Ehrhart et al., 2009, Romero et al., 2012). The original creators of TIPI have noted that the scale was designed to maximize the content validity and breadth of coverage in a few adjectives which would cost them the standards of internal consistency. Nevertheless, future studies could use longer versions of the big five personality factors scales to rule out measurement errors of short scales. Moreover, the internal consistency of the psychopathy subscale in the dark triad traits scale was very low. Therefore, we encourage future researchers to consider these issues in their work.

Finally, a methodological limitation of the current studies is their correlational nature which implies that one should be cautious in ascribing causality to our findings. Future studies should use experimental designs for investigating BJW's role in well-being, religiosity, and perceived threat of injustice. Although GBJW and PBJW are usually measured as traits, it is also possible to manipulate them (see Kogut, 2011).

\section{Conclusion}

This set of studies provided support for the BJW scales in a predominantly Muslim country. As in the case of the original, the two-factor model that includes GBJW and PBJW was proved to fit well. The validity of GBJW and PBJW scales was further investigated by examining the associations between these dimensions and self-esteem, life satisfaction, religiosity, big five personality factors, and dark triad traits. Consistent with the literature, GBJW and PBJW were correlated with these constructs with different strengths.

In order to provide additional support for the validity of the distinction between PBJW and GBJW, perceptions of injustice as kin favoritism were explored in two conditions. For the first condition in which individuals imagined other people as potential victims of injustice, the beliefs about the existence of justice in general were more important in how they perceived the outcomes of the scenarios than the belief in a 
personally just world. However, in the second condition in which they imagined themselves in the same scenarios, the belief about whether they are personally treated fairly by others were dominant predictors of their outcome perceptions. Furthermore, perceived moral and legal punishments partially explained the relationships between BJW and perceived intention of kin favoritism in both conditions. While replicating previous research on the role of BJW in risk perception and trust in others, we examined BJW's role in perceived threat of becoming a victim of injustice in the context of kin favoritism which is an overlooked and pervasive form of injustice. Future research should explore other aspects of kin favoritism, especially in societies with stronger family ties that suffer more from this type of injustice. 


\section{References}

Aghababaei, N., Mohammadtabar, S., \& Saffarinia, M. (2014). Dirty dozen vs. the h factor: Comparison of the dark triad and honesty-humility in prosociality, religiosity, and happiness. Personality and Individual Differences, 67, 6-10.

Akbari, M., Bahrami-Rad, D., Kimbrough, E. O., Romero, P. P., \& Alhosseini, S. (2020). An experimental study of kin and ethnic favoritism. Economic Inquiry, 58(4), 1795-1812.

Ames, D. R., Rose, P., \& Anderson, C. P. (2006). The npi-16 as a short measure of narcissism. Journal of research in personality, 40(4), 440-450.

Atari, M., \& Yaghoubirad, M. (2016). The big five personality dimensions and mental health: The mediating role of alexithymia. Asian journal of psychiatry, 24, 59-64.

Bai, B.-y., Liu, X.-x., \& Kou, Y. (2014). Belief in a just world lowers perceived intention of corruption: The mediating role of perceived punishment. PloS one, 9(5), e97075.

Bai, B.-y., Liu, X.-x., \& Kou, Y. (2016). Belief in a just world lowers bribery intention. Asian Journal of Social Psychology, 19(1), 66-75.

Bègue, L. (2002). Beliefs in justice and faith in people: Just world, religiosity and interpersonal trust. Personality and Individual Differences, 32(3), 375-382.

Bollmann, G., Krings, F., Maggiori, C., \& Rossier, J. (2015). Differential associations of personal and general just-world beliefs with the five-factor and the hexaco models of personality. Personality and Individual Differences, 87, 312-319.

Čábelková, I., \& Hanousek, J. (2004). The power of negative thinking: Corruption, perception and willingness to bribe in ukraine. Applied Economics, 36(4), 383-397.

Cubela Adoric, V., \& Kvartuc, T. (2007). Effects of mobbing on justice beliefs and adjustment. European psychologist, 12(4), 261-271.

Dalbert, C. (1999). The world is more just for me than generally: About the personal belief in a just world scale's validity. Social justice research, 12(2), 79-98. 
Dalbert, C., Montada, L., \& Schmitt, M. (1987). Glaube an eine gerechte welt als motiv: Validierungskorrelate zweier skalen. Psychologische Beitrage.

Diener, E., Emmons, R. A., Larsen, R. J., \& Griffin, S. (1985). The satisfaction with life scale. Journal of personality assessment, 49(1), 71-75.

Donat, M., Dalbert, C., \& Kamble, S. V. (2014). Adolescents' cheating and delinquent behavior from a justice-psychological perspective: The role of teacher justice. European Journal of Psychology of Education, 29(4), 635-651.

Donat, M., Knigge, M., \& Dalbert, C. (2018). Being a good or a just teacher: Which experiences of teachers' behavior can be more predictive of school bullying? Aggressive behavior, 44(1), 29-39.

Donat, M., Peter, F., Dalbert, C., \& Kamble, S. V. (2016). The meaning of students' personal belief in a just world for positive and negative aspects of school-specific well-being. Social Justice Research, 29(1), 73-102.

Donat, M., Umlauft, S., Dalbert, C., \& Kamble, S. V. (2012). Belief in a just world, teacher justice, and bullying behavior. Aggressive Behavior, 38(3), 185-193.

Ehrhart, M. G., Ehrhart, K. H., Roesch, S. C., Chung-Herrera, B. G., Nadler, K., \& Bradshaw, K. (2009). Testing the latent factor structure and construct validity of the ten-item personality inventory. Personality and individual Differences, 47(8), 900-905.

Furnham, A. (2003). Belief in a just world: Research progress over the past decade. Personality and individual differences, 34(5), 795-817.

Gosling, S. D., Rentfrow, P. J., \& Swann Jr, W. B. (2003). A very brief measure of the big-five personality domains. Journal of Research in personality, 37(6), 504-528.

Hafer, C. L., \& Gosse, L. (2011). Predicting alternative strategies for preserving a belief in a just world: The case of repressive coping style. European Journal of Social Psychology, 41(6), 730-739. 
Hafizi, S., Memari, A. H., Pakrah, M., Mohebi, F., Saghazadeh, A., \& Koenig, H. G. (2013). The duke university religion index (durel): Validation and reliability of the farsi version. Psychological Reports, 112(1), 151-159.

Harvey, A. J., \& Callan, M. J. (2014). The role of religiosity in ultimate and immanent justice reasoning. Personality and Individual Differences, 56, 193-196.

Jonason, P. K., \& Webster, G. D. (2010). The dirty dozen: A concise measure of the dark triad. Psychological assessment, 22(2), 420.

Joshanloo, M., \& Afshari, S. (2011). Big five personality traits and self-esteem as predictors of life satisfaction in iranian muslim university students. Journal of Happiness Studies, 12(1), 105-113.

Kiral Ucar, G., Hasta, D., \& Malatyali, M. K. (2019). The mediating role of perceived control and hopelessness in the relation between personal belief in a just world and life satisfaction. Personality and Individual Differences, 143, 68-73.

Koenig, H., Parkerson Jr, G. R., \& Meador, K. G. (1997). Religion index for psychiatric research.

Kogut, T. (2011). Someone to blame: When identifying a victim decreases helping. Journal of Experimental Social Psychology, 47(4), 748-755.

Lerner, M. J. (1980). The belief in a just world. The belief in a just world (pp. 9-30). Springer.

Lipkus, I. M., Dalbert, C., \& Siegler, I. C. (1996). The importance of distinguishing the belief in a just world for self versus for others: Implications for psychological well-being. Personality and Social Psychology Bulletin, 22(7), 666-677.

Nartova-Bochaver, S., Donat, M., Astanina, N., \& Rüprich, C. (2018). Russian adaptations of general and personal belief in a just world scales: Validation and psychometric properties. Social Justice Research, 31(1), 61-84. 
Nartova-Bochaver, S., Donat, M., \& Rüprich, C. (2019). Subjective well-being from a just-world perspective: A multi-dimensional approach in a student sample. Frontiers in psychology, 10, 1739.

Nudelman, G. (2013). The belief in a just world and personality: A meta-analysis. Social Justice Research, 26(2), 105-119.

Nudelman, G., \& Otto, K. (2021). Personal belief in a just world and conscientiousness: A meta-analysis, facet-level examination, and mediation model. British Journal of Psychology, 112(1), 92-119.

Paulhus, D. L., \& Williams, K. M. (2002). The dark triad of personality: Narcissism, machiavellianism, and psychopathy. Journal of research in personality, 36(6), $556-563$.

Romero, E., Villar, P., Gómez-Fraguela, J. A., \& López-Romero, L. (2012). Measuring personality traits with ultra-short scales: A study of the ten item personality inventory (tipi) in a spanish sample. Personality and Individual Differences, 53(3), 289-293.

Rosenberg, M. (1965). Rosenberg self-esteem scale (rse). Acceptance and commitment therapy. Measures package, 61(52), 18.

Shapurian, R., Hojat, M., \& Nayerahmadi, H. (1987). Psychometric characteristics and dimensionality of a persian version of rosenberg self-esteem scale. Perceptual and Motor Skills, 65(1), 27-34.

Shepperd, J. A., Klein, W. M., Waters, E. A., \& Weinstein, N. D. (2013). Taking stock of unrealistic optimism. Perspectives on Psychological Science, 8(4), 395-411.

Strelan, P., \& Callisto, J. (2020). Good outcomes are more likely for me than you - especially in a just world. Personality and Individual Differences, 163, 110033.

Strelan, P., \& Sutton, R. M. (2011). When just-world beliefs promote and when they inhibit forgiveness. Personality and Individual Differences, 50(2), 163-168. 
Sutton, R. M., \& Douglas, K. M. (2005). Justice for all, or just for me? more evidence of the importance of the self-other distinction in just-world beliefs. Personality and Individual Differences, $39(3), 637-645$.

Tatsi, S., \& Panagiotopoulou, P. (2021). Personal and general belief in a just world and self-esteem in primary school students. Current Psychology, 1-10.

White, C. J., Norenzayan, A., \& Schaller, M. (2019). The content and correlates of belief in karma across cultures. Personality and Social Psychology Bulletin, 45(8), 1184-1201.

Yu, G., Zhao, F., Wang, H., \& Li, S. (2020). Subjective social class and distrust among chinese college students: The mediating roles of relative deprivation and belief in a just world. Current Psychology, 39(6), 2221-2230. 


\section{Table 1}

Principal component analysis results and descriptive statistics of GBJW and PBJW

Item

$\mathrm{l}_{1} \quad \mathrm{l}_{2} \quad M \quad S D$

General Belief in a Just World

GBJW1: I think basically the world is a just place.

$\begin{array}{llll}.762 & .068 & 2.97 & 2.04\end{array}$

GBJW2: I believe that, by and large, people get what they deserve.

$\begin{array}{llll}.714 & .136 & 4.24 & 1.97\end{array}$

GBJW3: I am confident that justice always prevails over injustice.

$\begin{array}{llll}.859 & -.018 & 3.63 & 2.12\end{array}$

GBJW4: I am convinced that in the long run people will be

$.942 \quad-.166 \quad 4.18 \quad 1.97$ compensated for injustices.

GBJW5: I firmly believe that injustices in all areas of life

$\begin{array}{llll}.748 & .076 & 2.41 & 1.61\end{array}$

(e.g., professional, family, politic) are the exception rather than the rule.

GBJW6: I think people try to be fair when making important decisions.

$\begin{array}{llll}.543 & .100 & 3.59 & 1.56\end{array}$

\section{Personal Belief in a Just World}

PBJW1: I believe that, by and large, I deserve what happens to me.

$\begin{array}{llll}.073 & .641 & 4.91 & 1.84\end{array}$

PBJW2: I am usually treated fairly.

$\begin{array}{llll}-.192 & .851 & 4.44 & 1.69\end{array}$

PBJW3: I believe that I usually get what I deserve.

$\begin{array}{llll}.170 & .562 & 5.26 & 1.69\end{array}$

PBJW4: I believe that most of the things that happen in my life are fair. $\quad \begin{array}{llll}.108 & .787 & 4.33 & 1.81\end{array}$

PBJW5: In my life injustice is the exception rather than the rule. $\quad \begin{array}{llll}\text { P. } & \text {. } & 32.54 & 132\end{array}$

PBJW6: Overall, events in my life are just.

$\begin{array}{llll}-.032 & .841 & 4.15 & 1.73\end{array}$

PBJW7: I think that important decisions that are made concerning me $\quad .041 \quad .752 \quad 4.04 \quad 1.62$ are usually just.

Note: GBJW general belief in a just world, PBJW personal belief in a just world. 


\section{Table 2}

Correlations among Study 1 variables

\begin{tabular}{llllllllllllllll}
\hline Variables & 1 & 2 & 3 & 4 & 5 & 6 & 7 & 8 & 9 & 10 & 11 & 12 & 13 & 14 \\
\hline
\end{tabular}

1. GBJW

2. PBJW

3. Extraversion $.57^{* * *}$

4. Agreeableness

$$
.20^{* * *} \cdot 18^{* * *}
$$

5. Conscientiousness

$$
\begin{array}{lll}
.08 & .05 & -.05
\end{array}
$$

6. Emotional Stability

$.19^{* * *} .16^{* * *}-.06 \quad .14^{* *}$

7. Openness

$.16^{* * *} .24^{* * *}-.06 \quad-.01 \quad .19^{* * *}$

8. Machiavellianism

$\begin{array}{llllll}-.07 & -.02 & .05 & -.02 & .01 & .13^{* *}\end{array}$

9. Psychopathy

$\begin{array}{lllll}.01 & -.01 & .01 & -.27^{* * *}-.24^{* * *}-.06 & -.07\end{array}$

10. Narcissism

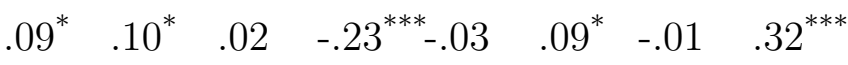

11. Self-Esteem

$.02 \quad .06 \quad .09^{*}-.09^{*}-.15^{* *}-.16^{* * *}-.01 \quad .38^{* * *} .13^{* *}$

12. Life Satisfaction

$.29^{* * *} .47^{* * *} .23^{* * *}-.01 \quad .34^{* * *} .33^{* * *} .17^{* * *}-.17^{* * *}-.09^{*}-.15^{* *}$

13. Religiosity

$.45^{* * *} .60^{* * *} .28^{* * *} .11^{*} \quad .28^{* * *} .19^{* * *}-.01 \quad-.05 \quad .12^{* *}-.10^{*} \quad .58^{* * *}$

14. Age $.60^{* * *} .36^{* * *} .16^{* *} .18^{* * *} .18^{* * *} .03 \quad-.26^{* * *}-.11^{* *}-.06 \quad-.05 \quad .18^{* * *} .34^{* * *}$

15. Gender $.20^{* * *} .02 \quad .14^{* *} \quad .09 \quad .22^{* * *} .04 \quad-.11^{*}-.13^{* *}-.13^{* *}-.28^{* * *} \cdot 16^{* * *} .17^{* * *} .28^{* * *}$ $\begin{array}{lllllllllll}.03 & .02 & .06 & .29^{* * *} .10^{*} & -.15^{* *} .01 & -.31^{* * *}-.06 & -.05 & -.01 & .07 & .14^{* *}-.03\end{array}$

Note: Gender was dummy-coded as 0 for male and 1 for female. GBJW general belief in a just world, PBJW personal belief in a just world. ${ }^{*} p<.05 .{ }^{* *} p<.01 .{ }^{* * *} p<.001$. 


\section{Table 3}

Correlations among Study 2 variables

\begin{tabular}{lccccccccc}
\hline Variables & $M$ & $S D$ & 1 & 2 & 3 & 4 & 5 & 6 & 7 \\
\hline 1. GBJW & 3.30 & 1.54 & & & & & & \\
2. PBJW & 4.26 & 1.29 & $.55^{* * *}$ & & & & & \\
3. Perceived Punishment & 4.24 & 1.53 & $.37^{* * *}$ & & & & & & \\
4. Perceived Intention of Kin Favoritism & 6.26 & 1.27 & $-.42^{* * *}-.36^{* *}$ & & & & & \\
5. Age & 32.57 & 7.39 & -.01 & -.09 & .05 & -.13 & & & \\
6. Gender & .57 & .49 & .11 & -.08 & .15 & .05 & .14 \\
\hline
\end{tabular}

Note: Gender was dummy-coded as 0 for male and 1 for female. GBJW general belief in a just world, PBJW personal belief in a just world.

${ }^{*} p<.05 .{ }^{* *} p<.01 .{ }^{* * *} p<.001$. 


\section{Table 4}

The results of hierarchical regression for predicting the threat of kin favoritism when the individuals are potential victims of injustice

\begin{tabular}{|c|c|c|c|c|c|c|}
\hline \multirow[b]{2}{*}{ Variables } & \multicolumn{2}{|c|}{ Model 1} & \multicolumn{2}{|c|}{ Model 2} & \multicolumn{2}{|c|}{ Model 3} \\
\hline & $\beta$ & $t$ & $\beta$ & $t$ & $\beta$ & $t$ \\
\hline Age & -.15 & -1.32 & -.17 & -1.74 & -.15 & -1.72 \\
\hline Gender & .07 & .62 & .09 & .92 & .14 & 1.54 \\
\hline GBJW & & & -.33 & $-2.71^{* *}$ & -.16 & -1.47 \\
\hline PBJW & & & -.18 & -1.54 & -.19 & -1.76 \\
\hline Perceived Likelihood of Punishment & & & & & -.45 & $-4.71^{* *}$ \\
\hline$R^{2}$ & .02 & & .23 & & .41 & \\
\hline$F$ & .97 & & $5.92^{*}$ & & $10.50^{*}$ & \\
\hline$\Delta R^{2}$ & & & .21 & & .18 & \\
\hline
\end{tabular}

Note: Gender was dummy-coded as 0 for male and 1 for female. GBJW general belief in a just world, PBJW personal belief in a just world.

${ }^{*} p<.05 .{ }^{* *} p<.01 .{ }^{* * *} p<.001$. 


\section{Table 5}

Correlations among Study 3 variables

\begin{tabular}{|c|c|c|c|c|c|c|c|c|c|}
\hline Variables & $M$ & $S D$ & 1 & 2 & 3 & 4 & 5 & 6 & 7 \\
\hline 1. GBJW & 3.50 & 1.43 & & & & & & & \\
\hline 2. PBJW & 4.40 & 1.29 & $.50^{* * *}$ & & & & & & \\
\hline 3. Perceived Punishment & 4.69 & 1.61 & $.28^{*}$ & $.40^{* * *}$ & & & & & \\
\hline 4. Perceived Intention of Kin Favoritism & 5.55 & 1.67 & $-.27^{*}$ & $-.35^{* *}$ & $-.44^{* * *}$ & & & & \\
\hline 5. Age & 30.52 & 5.07 & .16 & -.06 & -.02 & -.02 & & & \\
\hline 6. Gender & .54 & .50 & .20 & -.07 & -.03 & .01 & .20 & & \\
\hline
\end{tabular}

Note: Gender was dummy-coded as 0 for male and 1 for female. GBJW general belief in a just world, PBJW personal belief in a just world.

${ }^{*} p<.05 .{ }^{* *} p<.01 .{ }^{* * *} p<.001$. 


\section{Table 6}

The results of hierarchical regression for predicting the intention of kin favoritism when individuals are observers of a potential injustice

\begin{tabular}{|c|c|c|c|c|c|c|}
\hline \multirow[b]{2}{*}{ Variables } & \multicolumn{2}{|c|}{ Model 1} & \multicolumn{2}{|c|}{ Model 2} & \multicolumn{2}{|c|}{ Model 3} \\
\hline & $\beta$ & $t$ & $\beta$ & $t$ & $\beta$ & $\mathrm{t}$ \\
\hline Age & -.03 & -.24 & -.02 & -.22 & -.03 & -.28 \\
\hline Gender & .02 & .18 & .02 & .22 & .16 & 0.14 \\
\hline GBJW & & & -.13 & -.93 & -.08 & -.66 \\
\hline PBJW & & & -.28 & $-2.10^{*}$ & -.16 & -1.21 \\
\hline Perceived Punishment & & & & & -.35 & $-2.99^{* *}$ \\
\hline$R^{2}$ & .01 & & .13 & & .24 & \\
\hline$F$ & .04 & & $2.66^{*}$ & & $4.18^{* *}$ & \\
\hline$\Delta R^{2}$ & & & .12 & & .11 & \\
\hline
\end{tabular}

Note: Gender was dummy-coded as 0 for male and 1 for female. GBJW general belief in a just world, PBJW personal belief in a just world.

${ }^{*} p<.05 .{ }^{* *} p<.01 .{ }^{* * *} p<.001$. 


\section{Appendix}

\section{Kin Favoritism Scenarios}

1. You are a PhD student in one of Iran's prestigious universities. Your supervisor has been given an opportunity to choose a student to study abroad as a visiting scholar for 6 months. He/she must choose among you and other students under his/her supervision. You are the one with the best GPA and resume but one of the other students is a relative of your supervisor. The supervisor clearly knows that any kind of discrimination is against the university's rules and regulations.

2. You are a film director and the movie you have recently made is going to be reviewed in an influential film magazine. This week, another movie with a much lower quality is going to be evaluated beside yours, and one of the movies will be chosen and featured as the "best movie of the week". Various critics work under the supervision of the chief editor of the magazine. Coincidentally, the critic who is in charge of this week's review process is a relative of the other movie's director. This critic clearly knows that discrimination and choosing a movie based on anything other than cinematic standards is against the magazine's rules and regulations.

3. You have taken your lawsuit to a local court. After reviewing the case carefully, your lawyer has assured you that you have the upper hand, and the judge will give a verdict in your favor. Coincidentally, the judge that is assigned to your case is a relative of the other party. All the judges in the courthouse work under the supervision of the Department of Justice. The judge clearly knows that discrimination and giving a verdict based on anything other than the case material is against the law.

4. You have been chosen as one of the two final candidates for a government job position. Considering the resumes, you are clearly better qualified for the job than the other candidate. A recruiter who works under the supervision of higher ranks has to do an interview with you and the other candidate to decide who gets the job. Coincidentally, the recruiter turned out to be a relative of the other candidate. This recruiter knows that 
discriminating between candidates and not choosing the most qualified person is against the rules.

5. You have had a car accident in a secluded area of the city and both cars have had minimal damage. The other driver is clearly to blame for this accident. A police officer arrives and investigates the scene of the accident. Coincidentally, the officer called for this accident is a relative of the other driver. This police officer clearly knows discrimination for any reason is against the law. 